\title{
Quantum Entanglement: Where Dark Energy and Negative Gravity plus Accelerated Expansion of the Universe Comes from
}

\author{
Mohamed S. El Naschie \\ Department of Physics, Alexandria University, Alexandria, Egypt \\ Email: chaossf@aol.com
}

Received March 11, 2013; revised April 27, 2013; accepted May 16, 2013

Copyright (C) 2013 Mohamed S. El Naschie. This is an open access article distributed under the Creative Commons Attribution License, which permits unrestricted use, distribution, and reproduction in any medium, provided the original work is properly cited.

\begin{abstract}
Dark energy is shown to be the absolute value of the negative kinetic energy of the halo-like quantum wave modeled mathematically by the empty set in a five dimensional Kaluza-Klein (K-K) spacetime. Ordinary or position energy of the particle on the other hand is the dual of dark energy and is contained in the dynamic of the quantum particle modeled by the zero set in the same five dimensional K-K spacetime. The sum of both dark energy of the wave and the ordinary energy of the particle is exactly equal to the energy given by the well known formula of Einstein $E=m c^{2}$ which is set in a four dimensional spacetime. Various interpretations of the results are presented and discussed based on the three fundamental energy density equations developed. In particular $E$ (ordinary) $=\left(\phi^{5} / 2\right) m c^{2} \quad m c^{2} / 22$ where $E$ is the energy, $m$ is the mass and $c$ is the speed of light, $\phi^{5}$ is Hardy's quantum entanglement and $\phi=2 /(1+\sqrt{5})$ gives results in complete agreement with the cosmological measurements of WMAP and Supernova. On the other hand $E($ dark $)=\left|-m c^{2}(21 / 22)\right|$ gives an intuitive explanation of negative gravity and the observed increased rate of cosmic expansion. Adding $E$ (ordinary) to $E$ (dark) one finds $E=\left(m c^{2} / 22\right)+m c^{2}(21 / 22)=m c^{2}$ which as we mentioned above is Einstein's famous relativity formula. We conclude that similar to the fact that the quantum wave interpreted generally as probability wave which is devoid of ordinary energy decides upon the location of a quantum particle, it also exerts a negative gravity effect on the cosmic scale of our clopen, i.e. closed and open universe. Analysis and conclusions are framed in a reader friendly manner in Figures 1-14 with detailed commentary.
\end{abstract}

Keywords: Dark Kinetic Energy of the Quantum Wave; Ordinary Position Energy of the Quantum Particle; Anti Gravity; Negative Curvature; Collapse of the Hawking-Hartle Quantum Wave of the Universe; Revising Einstein's Relativity; Quantum Gravity

\section{Introduction}

We utilize the wave-particle duality [1,2] and quantum entanglement [2-8] as fundamental features of quantum mechanics $[1,8]$ as well as classical field, quantum set theory [3] and transfinite quantization [4-44] to develop an effective quantum gravity energy theory $[1-8]$ that can convincingly resolve the problem of the accelerated rate of cosmic expansion and the missing dark energy of the cosmos and at the same time agrees with the accurate WMAP and Supernova cosmic energy density measurements and analysis [9-18]. It turns out that ordinary energy is intimately related to the position energy of a generic quantum particle $[9,20]$ in five dimensional Kaluza-
Klein spacetime $[2,4,6]$ and amounts to

$$
E_{1}=\left(\phi^{5} / 2\right) m c^{2} \quad m c^{2} / 22
$$

where $E$ is the energy, $m$ is the mass, $c$ is the speed of light, $\phi^{5}$ is Hardy's quantum entanglement and $\phi=2 /(\sqrt{5}+1) \quad[12,17,18]$ (see Figure 1). Dark energy on the other hand was found to be a hitherto unknown and unsuspected halo-like feature of a generic quantum probability wave with a negative kinetic energy inducing negative gravity (see Figure 2). Thus a certain broken symmetry governs particles and waves from a set theoretical perspective $[1,8]$. In short the quantum particle is the ultimate source of ordinary energy and contributes to 
Ordinary positive energy of the quantum particle and negative dark energy of the quantum wave are derived from the zero set and the empty set respectively via Kaluza-Klein $\mathrm{D}=5$

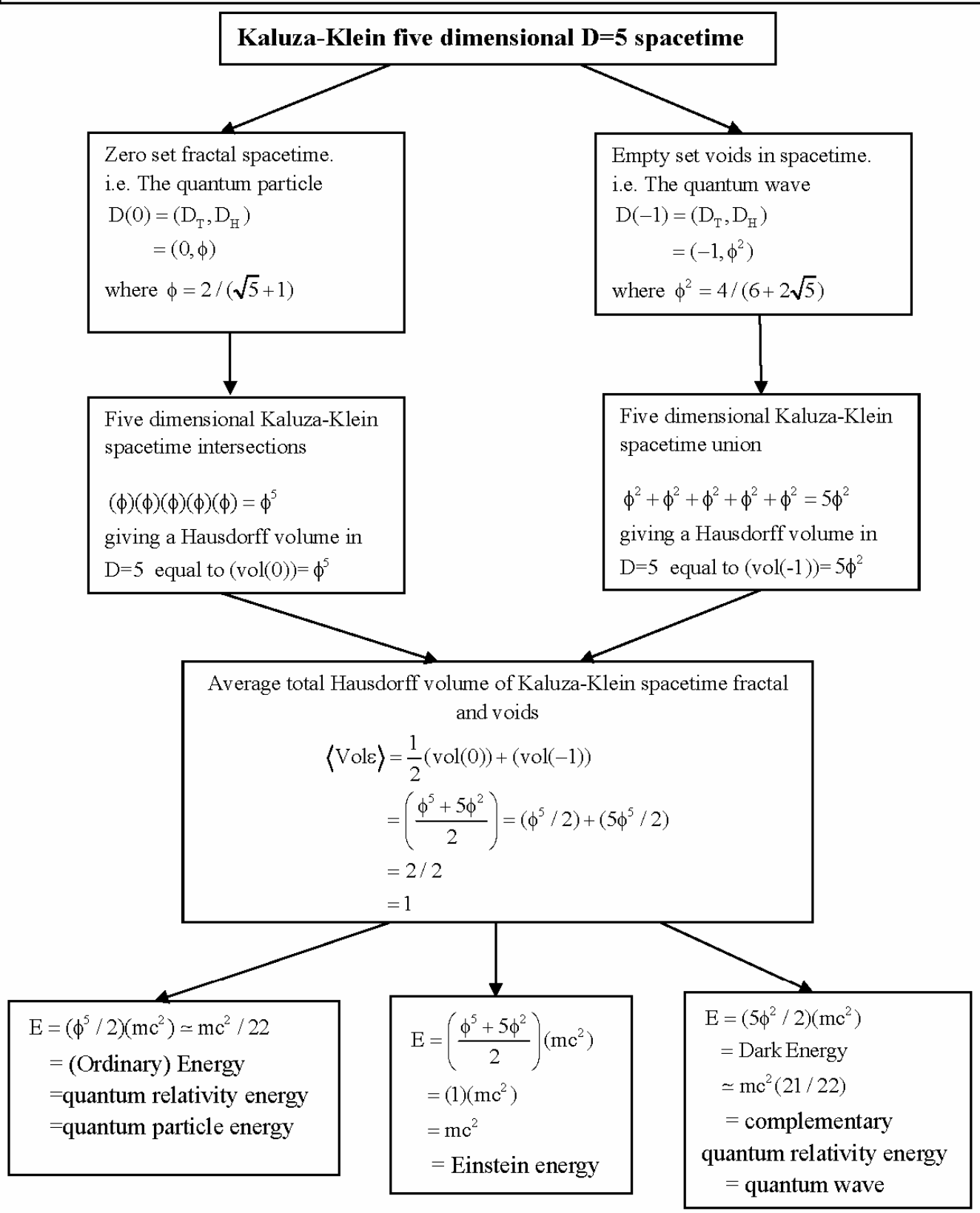

Note that in all above explanations we let the velocity $v$ tends simply to that of the velocity of light $(\mathrm{v} \rightarrow \mathrm{c}$ ) and the division by 2 is due to fractal averaging and not due using Newton's Kinetic energy $\mathrm{E}_{\mathrm{N}}=\frac{1}{2} \mathrm{mv}^{2}$. There is thus another interpretation where we use $\mathrm{E}_{\mathrm{N}}$ and the transformation $\mathbf{v} \rightarrow \phi c$ and $m \rightarrow \phi^{3} \mathrm{~m}$ for ordinary energy for quantum particle and $\mathrm{m} \rightarrow 5 \mathrm{~m}$ for dark energy of quantum wave. Finally to find the total energy of Einstein we use $\mathrm{m} \rightarrow\left(5+\phi^{3}\right) \mathrm{m}$ and $\mathrm{v} \rightarrow \phi \mathrm{c}$ where $5+\phi^{3}$ is the dimension of a fractal Kaluza-Klein spacetime [5] for which D is not 5 but $5+\phi^{3}$. 


\section{Dark Energy, Ordinary Energy and Einstein Energy in a nutshell}

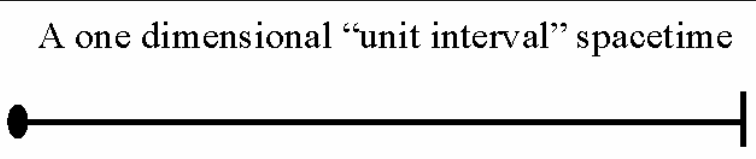

$\mathrm{D}($ Topological $)=\mathrm{D}($ Hausdorff $)=1$

We construct a cantor set from the one dimensional clopen i.e. closed and open spacetime interval

.......... $\cdot$ :

Uncountably infinitely many points

$\mathrm{D}($ Topological $)=\mathrm{D}($ Point $)=0$

$\mathrm{D}($ Hausdorff $)=\frac{\sqrt{5}-1}{2}=\phi$

Lifting $\phi$ by intersection i.e. multiplication theorem to a 5 dimensional Kaluza-Klein spacetime, we find a geometrical density or Hausdorff volume $\operatorname{vol}_{\mathrm{H}}=(\phi)^{5}$

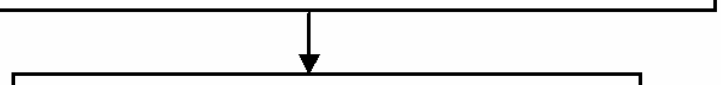

Newton Kinetic energy

$\mathrm{E}=\frac{1}{2} m v^{2}$ for $\mathrm{Vol}_{\mathrm{H}}=\phi^{5}$ is thus:

$\mathrm{E}_{1}=\left(\phi^{5} / 2\right) \mathrm{m}(\mathrm{v} \rightarrow \mathrm{c})^{2}$

$=\mathrm{mc}^{2} / 22$

$=$ Energy of the quantum particle

$=$ Ordinary Energy
The dimension of the random empty gaps left over from the construction of the Cantor set is a countablely infinitely many gaps given by $\mathrm{D}($ Topological $)=\mathrm{D}($ Empty set $)=-1$

$\mathrm{D}$ (Hausdorff) $=\mathrm{D}$ (unit interval) $-(\mathrm{D}=\phi)$

$$
\begin{aligned}
& =1-\phi \\
& =\phi^{2}
\end{aligned}
$$

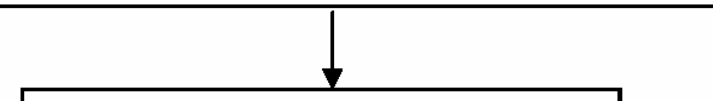

Lifting $\phi^{2}$ additively (union operation) to 5 dimensional Kaluza-Klein spacetime we find a Hausdorff volume vol $_{\mathrm{H}}=5 \phi^{2}$

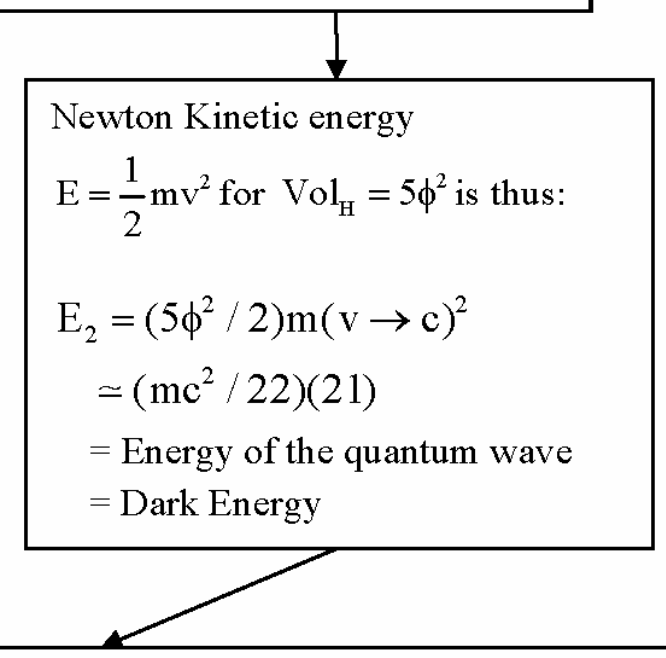

Adding $E_{1}$ of the quantum particle to $E_{2}$ of the quantum wave we find

$$
E=\left(\phi^{5}+\phi^{2}\right)\left(\frac{1}{2}\right) m c^{2}=m c^{2}=E(\text { Einstein })
$$

Figure 1. (a) The set theoretical ordinary energy-dark energy duality; (b) The quintessence of dark energy from the topology of the cantor set. 
about $(100) \times(1 / 22) \quad 4.5$ percent of the total energy of the cosmos $[18,19]$ (see Figures 1-12). In turn the quantum particle is modeled by the zero set represented dimensionally by von Neumann-Connes dimensional function $[8,44]$ of a Penrose quasicrystal fractal tiling space [21-23]. The broken mirror image of the quantum particle is the halo-like quantum probability wave which is the source of a negative energy anti-gravity (see Figures 9 and 11) stemming from modeling the quantum wave via the empty set of the same Penrose quasicrystals fractal tiling $[21-23,30-34,44]$. Subsequently the energy of the quantum wave was found to be $[12,18]$

$$
E_{2}=\left(5 \phi^{2} / 2\right) m c^{2} \quad m c^{2}(21 / 22)
$$

It was then found to the Author's delight that the sum of ordinary energy of the particle and absolute value of the negative dark energy of the wave added together is exactly the energy predicted by Einstein's formula [2,3, 17]

$$
E=E_{1}+E_{2}=\left[\left(\phi^{5}\right)+\left(5 \phi^{2}\right)\right]\left(\frac{1}{2}\right) m c^{2}=m c^{2} .
$$

Our most important conclusion in this work is that Einstein's $E=m c^{2}$ gives the correct total energy formula for an infinitely large Cantor set-like clopen i.e. closed and open universe [24-29] but it does not distinguish between ordinary energy and negative dark energy of a basically fractal cosmos. Noting that measurement leads to quantum wave collapse, it is completely normal that we cannot measure dark energy using present day technology. In the following sections we will attempt to explain more accurately but concisely the points transacted above. For a quick overview, the entire analysis and results are summarized in Figures 1-3, while valuable details and additional information are presented in Figures 4-12. In Figures 13 and 14 we give a naive yet very instructive geometrical visualization and interpretation of the wave-particle duality in five dimensional Kaluza-Klein spacetime.

\section{Basic Concept}

\subsection{Ordinary Energy, the Zero Set and the Quantum Particle}

Ordinary energy of the universe is directly proportional to the fractal nature of spacetime [4-12,30-34]. More accurately it is the multiplicative hyper Hausdorff measure or volume of the zero set i.e. the quantum particle in a five dimensional Kaluza-Klein universe $(D=5)$. The zero set which possessrs the cardinality of the continuum is fixed using the Bi-dimensions formula [8]

$$
D=\left(D_{T}, D_{H}\right)
$$

i.e. the topological dimension $D_{T}$ and the Hausdorff dimension $D_{H}$ so that we find

$$
D_{O}=(o ; \phi)
$$

where $\phi=(\sqrt{5}-1) / 2$ as in von Neumann-Connes' non-commutative geometry $[8,22,33]$ and its well known dimen dimensional function when applied to Penrose fractal clopen i.e. closed and open universe tiling $[21,44]$.

\subsection{Dark Energy, the Empty Set and the Quantum Wave}

Dark energy on the other hand is given by the dual additive hyper Hausdorff measure or volume of the empty set i.e. quantum wave in $D=5$ Kaluza-Klein spacetime $[4,6]$. Thus we start from $D(-1)$ which is the empty set given by [8]

$$
D_{-1}=\left(-1, \phi^{2}\right)
$$

i.e. $D_{T}=-1$ and $D_{H}=\phi^{2}$ for $D_{-1}[8,44]$.

\subsection{The Hausdorff "Volume" of the Zero Set in Five Dimensional Space and the Quantum Particle}

For the hyper Hausdorff volume of the fractal zero set of ordinary energy [8], we apply the intersection rule of sets in 5 Kaluza-Klein dimensions and find (see Figures 1(a) and (b))

$$
\operatorname{Vol}(5 D \text { Zero set })=(\phi)(\phi)(\phi)(\phi)(\phi)=\phi^{5} .
$$

In a sense this Hausdorff measure is the "volume" or the energy density of a quantum particle [8].

The volume $\phi^{5}$ could be naively interpreted geometrically as the generalization of the volume of a cube to a pseudo 5D cube with each side being of a length equal to $\phi$. (See Figures 13 and 14)

\subsection{The Hausdorff "Volume" of the Empty Set in Five Dimensional Space and the Quantum Wave}

On the other hand for the additive Hausdorff "volume" which is equal the length of the empty set $[22,33]$ of dark energy we have the addition or union rule of sets in 5 K-K dimension and one finds (see Figures 1(a) and (b))

$$
\begin{aligned}
& \operatorname{Vol}(5 D \text { Empty set) } \\
& =(\phi)^{2}+(\phi)^{2}+(\phi)^{2}+(\phi)^{2}+(\phi)^{2}=5 \phi^{2} .
\end{aligned}
$$

We used here the union of sets rather than the intersection because of the duality between particle and wave $[2,8]$ (see Figures 13 and 14). Consequently the Hausdorff measure of the empty set in K-K spacetime represents the energy density of a quantum wave which does not exist in the usual conventional quantum interpretation because a conventional quantum wave in orthodox quantum mechanics is a probability wave and has no 
Comparative analysis of ordinary quantum particle positive energy and dark quantum wave negative energy

The multiplicative Hausdorff volume $\operatorname{vol}(\mathrm{D}(0))$ of the zero set gives by $\mathrm{D}(0)=(0 ; \phi)$ which represents the quantum particle is

$\operatorname{vol}(\mathrm{D}(0))=(\phi)^{\mathrm{D}_{s}}$

Taking $\mathrm{D}_{\mathrm{s}}=5$ for a Kaluza-Klein spacetime one finds $\operatorname{vol}(\mathrm{D}(0))=\phi^{5}$.

Note that: $\phi^{5}$ where is the Hardy probability for quantum entanglement of two particles $\phi=(\sqrt{5}-1) / 2$.

Newton kinetic energy

$\mathrm{E}=\frac{1}{2} m v^{2}$ for $\operatorname{vol}(\mathrm{D}(0))$ and

$\mathrm{V} \rightarrow \mathrm{c}$ where $\mathrm{c}$ is the speed of

light becomes

$$
\begin{aligned}
\mathrm{E}_{1} & =\operatorname{vol}\left(\mathrm{D}_{0}\right)\left(\frac{1}{2} m \mathrm{c}^{2}\right) \\
& =\left(\phi^{5} / 2\right) m c^{2} \\
& \simeq m c^{2} / 22 \\
& =\text { ordinary Energy }
\end{aligned}
$$

which is the energy of a quantum particle
The dual to the Hausdorff volume of the zero set is the additive pseudo Hausdorff Volume of the empty set: $\operatorname{vol}(D(-1))=\left(-1, \phi^{2}\right)$ which represents the quantum wave. Consequently vol $(\mathrm{D}(-1))$ in five dimensions of Kaluza-Klein space is $\operatorname{vol}(D(-1))=(5)\left(\phi^{2}\right)$. Note that the empty set is the cobordism of the zero set. That means it is the surface of the quantum particle is the quantum wave. for there more $\left(5 \phi^{2}\right)$ could be given a negative sign because of the (-1) component of $D(-1)$ so that strictly speaking vol(D-1) is a negative volume in the measure theoretical meaning attached to a negative Menger-Uhryson deductive dimensional system.

$$
\begin{aligned}
& \text { Newton Kinetic energy } \\
& \begin{aligned}
\mathrm{E} & =\frac{1}{2} \mathrm{~m} v^{2} \text { for vol(D(-1)) and } \\
\mathrm{v} & \rightarrow \text { c becomes: } \\
\mathrm{E}_{2} & =\operatorname{vol}(\mathrm{D}(-1))\left(\frac{1}{2} \mathrm{mc}^{2}\right) \\
& =\left(5 \phi^{2} / 2\right)\left(\mathrm{mc}^{2}\right) \\
& \simeq\left(\mathrm{mc}^{2}\right)(21 / 22) \\
& =\text { Dark Energy }
\end{aligned}
\end{aligned}
$$

which is equal to the negative energy $(-1)\left(\mathrm{mc}^{2}\right)(21 / 22)$ producing negative gravity which is a halo energv caused bv the quantum wave

Figure 3. Comparative analysis of ordinary quantum particle positive energy and dark quantum wave negative energy. 


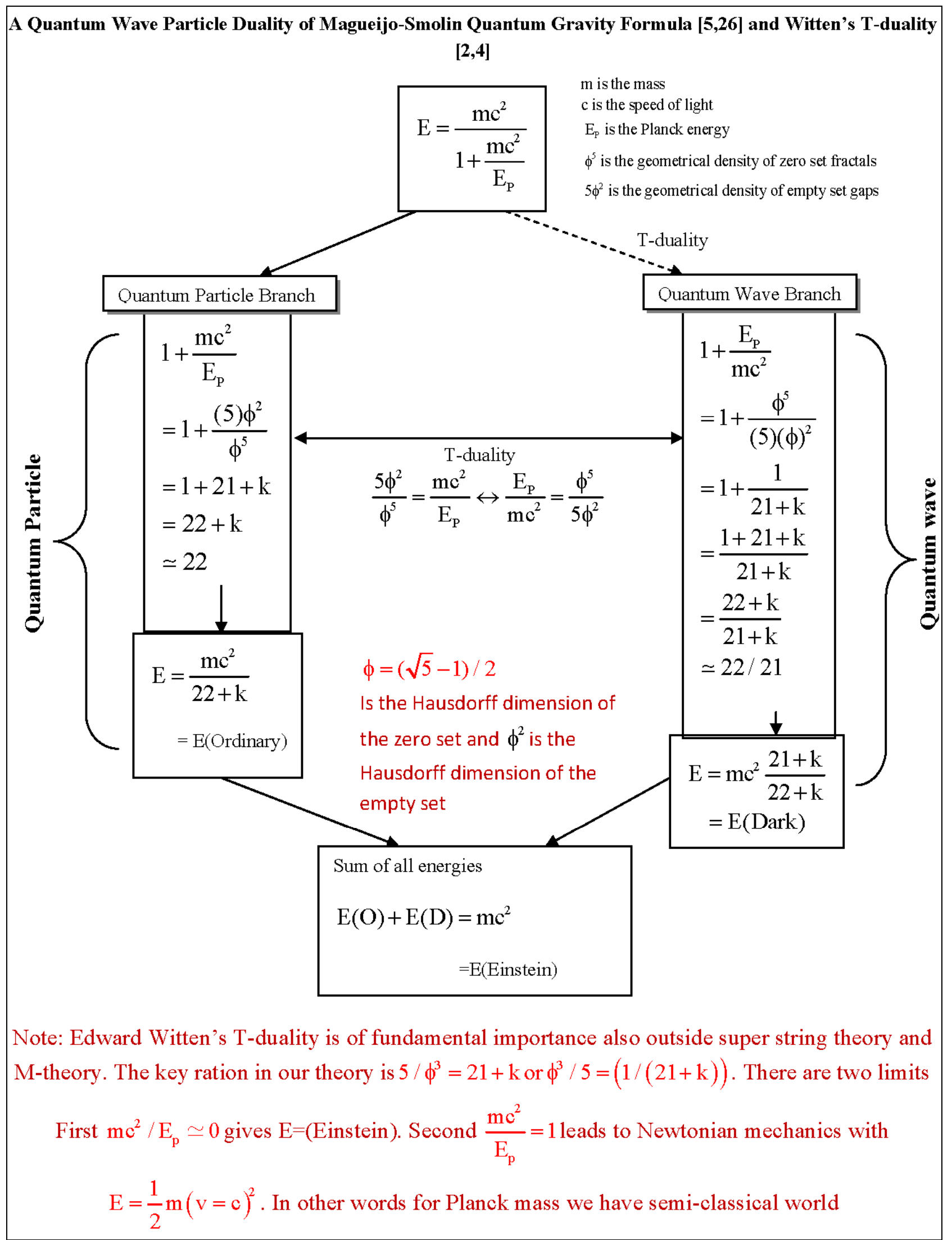

Figure 4. The universe as a fractal in infinity space. 


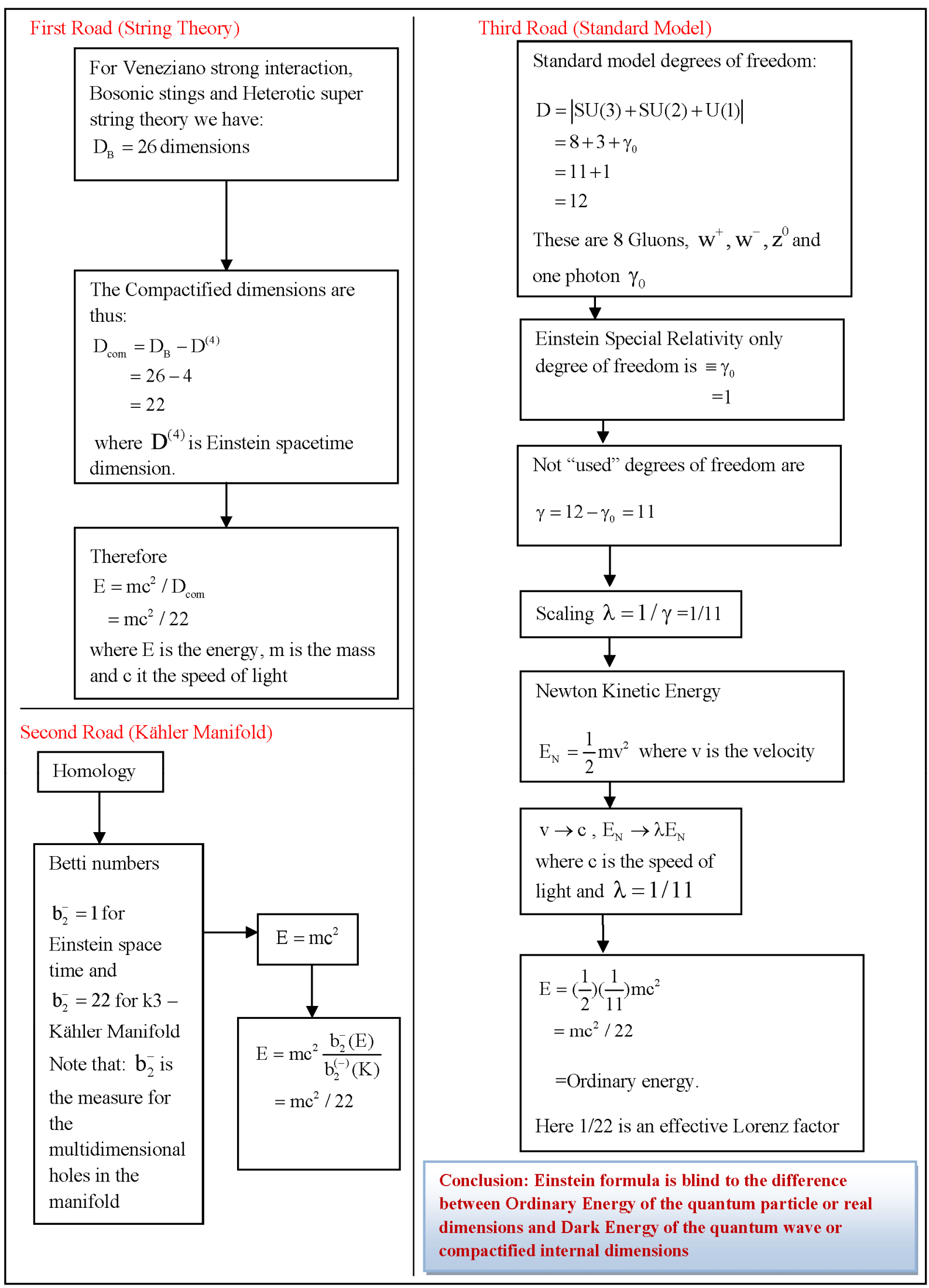

Figure 5. Special relativity with extra dimensional twist or all roads lead to Rome and all theories lead to $E \quad m c^{2} / 22$. 


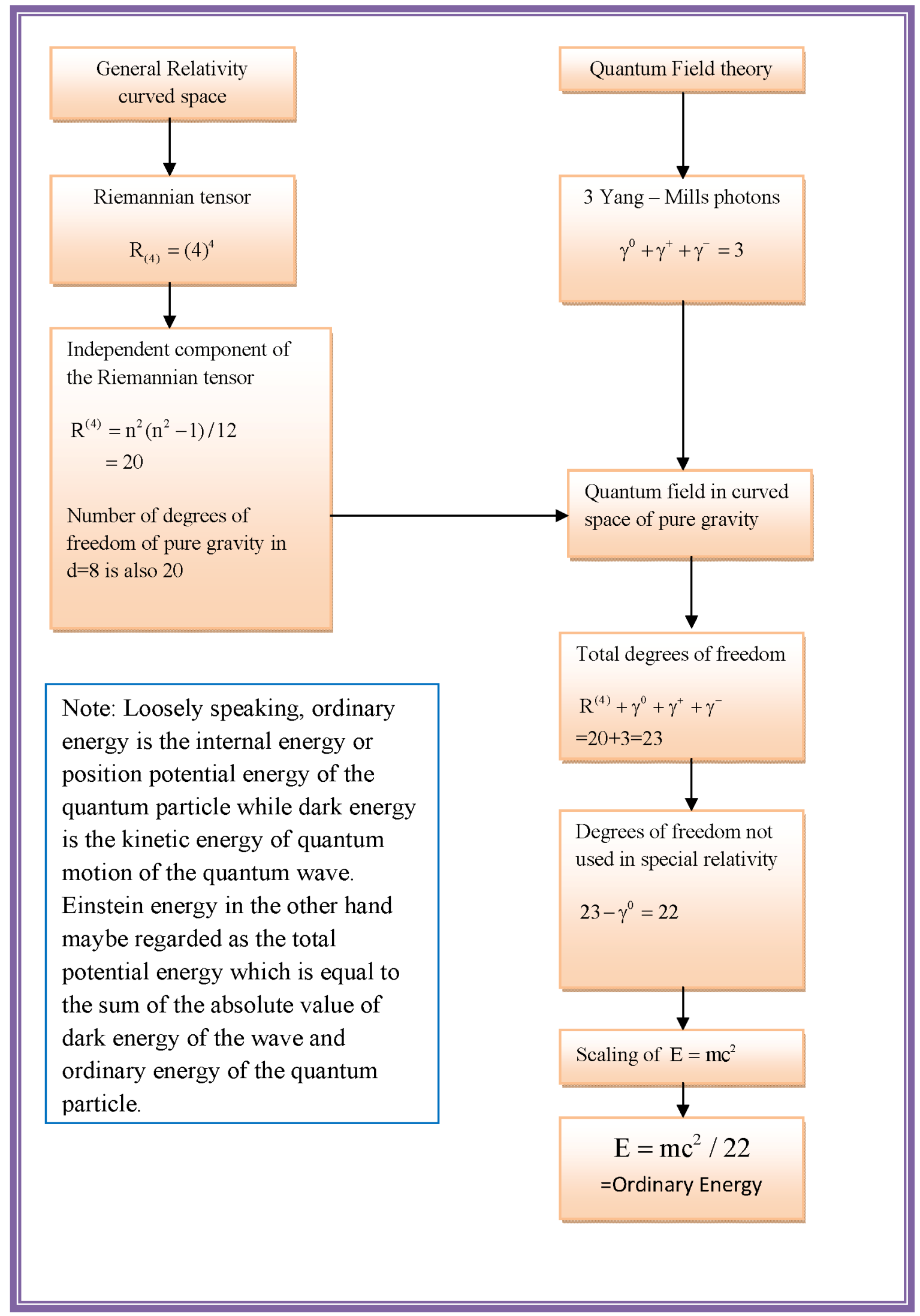

Figure 6. Yang-Mills for dark energy. Deriving $E \quad m c^{2} / 22$ from quantum field theory in curved spacetime. 


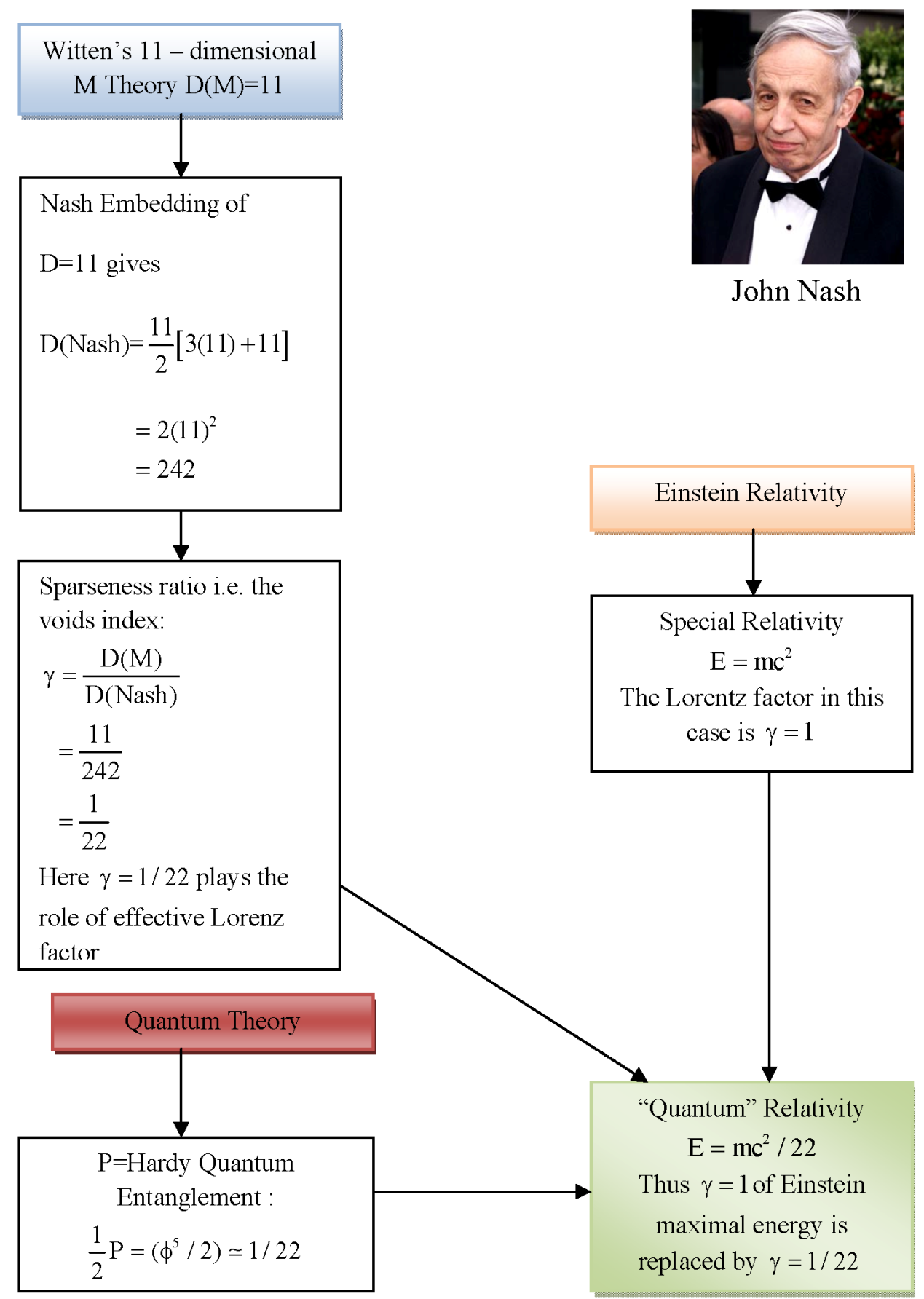

Note that: $\phi=(\sqrt{5}-1) / 2$ is the Hausdorff dimension of the zero set and $\mathrm{D}(\mathrm{Nash})=\frac{\mathrm{n}}{2}(3 \mathrm{n}+11)$ for compact manifold where $\mathrm{n}$ is the dimension of the manifold to be embedded.

Figure 7. A Beautiful mind for dark energy. A flow chart starting from Nash embedding and Witten's M-Theory explaining the logical connections and sequential deduction using Einstein relativity and leading to quantum relativity energy E $m c^{2} / 22$. 


\begin{tabular}{|c|c|}
\hline $\begin{array}{l}\text { Starting from Einstein's } 4 \mathrm{D} \text { Spacetime } \\
\text { and the } 26 \mathrm{D} \text { of Bosonic Strings we have }\end{array}$ & $\begin{array}{l}\text { Starting from Kaluza-Klein 5-D } \\
\text { Spacetime and the 26D of Bosonic } \\
\text { Strings we have }\end{array}$ \\
\hline $\begin{aligned} \mathrm{E}_{\mathrm{QR}} & =\frac{\mathrm{E}(\text { Einstein })}{\mathrm{D}^{(26)}-\mathrm{D}^{(4)}} \\
& =\frac{\mathrm{mc}^{2}}{\mathrm{D}^{(26)}-\mathrm{D}^{(4)}} \\
& =\frac{m c^{2}}{26-4} \\
& =\mathrm{mc}^{2} / 22\end{aligned}$ & $\begin{aligned} E & =\frac{E(\text { Einstein })}{1+\frac{m c^{2}}{E_{P}}} \\
& =\frac{\mathrm{mc}^{2}}{1+\left(\mathrm{D}^{(26)}-\mathrm{D}^{(5)}\right)} \\
& =\frac{\mathrm{mc}^{2}}{1+(26-5)} \\
& =\frac{\mathrm{mc}^{2}}{1+21} \\
& =m c^{2} / 22\end{aligned}$ \\
\hline \multicolumn{2}{|c|}{$\begin{array}{l}\text { Note that: } E_{\mathrm{P}} \text { is the Planck Energy } \mathrm{E}_{\mathrm{P}}=\sqrt{\frac{\mathrm{hc}^{5}}{\mathrm{G}}} \text {. Setting } \mathrm{h} / \mathrm{G}=\phi^{5} \text { and } \mathrm{C} \text { equal to } \\
\text { Sigalotti's critical value } \mathrm{c}=\phi=(\sqrt{5}-1) / 2 \text { we find that } \mathrm{E}_{\mathrm{P}}=\phi^{5} \text { and that the } \\
\text { rectangular area } \mathrm{E}_{\mathrm{P}}=\phi^{2} \phi^{3}=\phi^{5} \text { is Hardy's probability of quantum entanglement while } \\
\text { the triangular area } \frac{1}{2} \phi^{2} \phi^{3}=\frac{1}{2} \phi^{5} \text { is the } \gamma \text { factor in } \mathrm{E}_{\mathrm{QR}}=\left(\phi^{5} / 2\right) \mathrm{mc}^{2} \simeq \mathrm{mc}^{2} / 22 \text {. } \\
\text { Here } \phi^{2} \text { is the local entanglement and } \phi^{3} \text { is the global (counter factual) entanglement of } \\
\text { Hardy's quantum entanglement. }\end{array}$} \\
\hline
\end{tabular}

Figure 8. Bosonic strings interpretation of $E \quad m c^{2} / 22$ and varying speed of Light Theory $E=\frac{m c^{2}}{1+\frac{m c^{2}}{E_{P}}}$.

ordinary energy and collapses at measurement $[1,2]$. and collapse at measurement $[1,2]$. Again a possible naïvegeometrical interpretation of $5 \phi^{2}$ is being a one dimensional "surface" i.e. the circumference of a pentagon with each of the 5 sides being of a length equal to $\phi^{2}$ (see Figure 13). In particular the quantum wave determines the most probable location of a quantum particle but vanishes for a classically large object which is the contrary effect of negative gravity induced by the same wave i.e. anti gravity vanishes locally and exists only by accumulation at cosmic scales.

\subsection{The Average Hausdorff Volume of Quantum Particle and Wave as Total Relativity Energy}

From the above points (3) and (4) we see that the total geometrical density or pseudo Hausdorff "volume" is the sum of both the fractal part of spacetime $\phi^{5}$ and the voids "empty" part of spacetime $5 \phi^{2}$. This means (see Figures 1(a) and (b) as well as Figures 13 and 14)

$$
\operatorname{Vol}(\text { total })=\phi^{5}+5 \phi^{2}=2
$$

This is an extremely important result because an arithmetic average geometrical density or Hausdorff "dimensional volume" would give us

$$
\langle\operatorname{vol}(\text { total })\rangle=\left(\phi^{5}+5 \phi^{2}\right) / 2=1
$$

On the other hand the measure or "volume" or geometrical density of the unitarity set $D_{1}=(1 ; 1)$ is $\operatorname{vol}(D(1))=(1)^{5}=1$ and leads directly to Einstein's celebrated energy density formula $[2,5,17]$ (see Figures 1 and 2)

$$
E=m c^{2}(\operatorname{vol}(D(1)))=m c^{2},
$$

where $E$ is the energy, $m$ is the mass and $c$ is the speed of light $[2,3,12,17]$. 


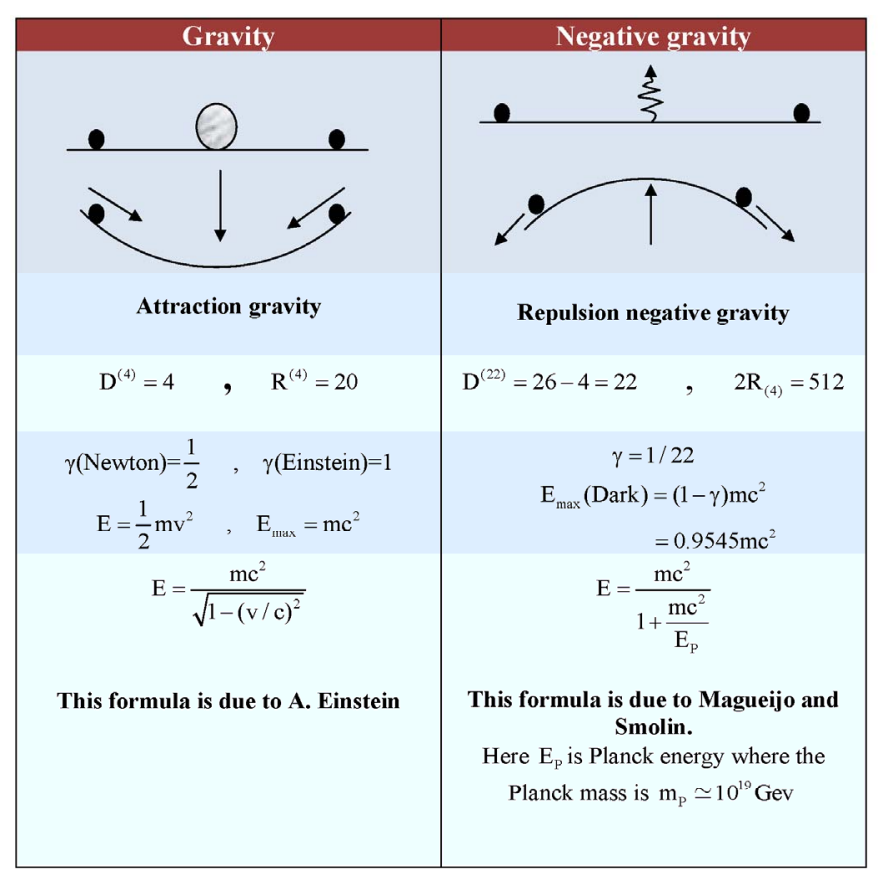

Here $\mathrm{D}^{(4)}=4$ is the dimension of Einstein's spacetime, $\mathrm{R}^{(4)}=20$ is the number of independent components of Riemann curvature tensor in dour dimensions and $\mathbf{R}_{(4)}=256$ is the number of all the components of the same curvature tensor [2], $v$ is the velocity and $\mathrm{c}$ is the speed of light.

(a)

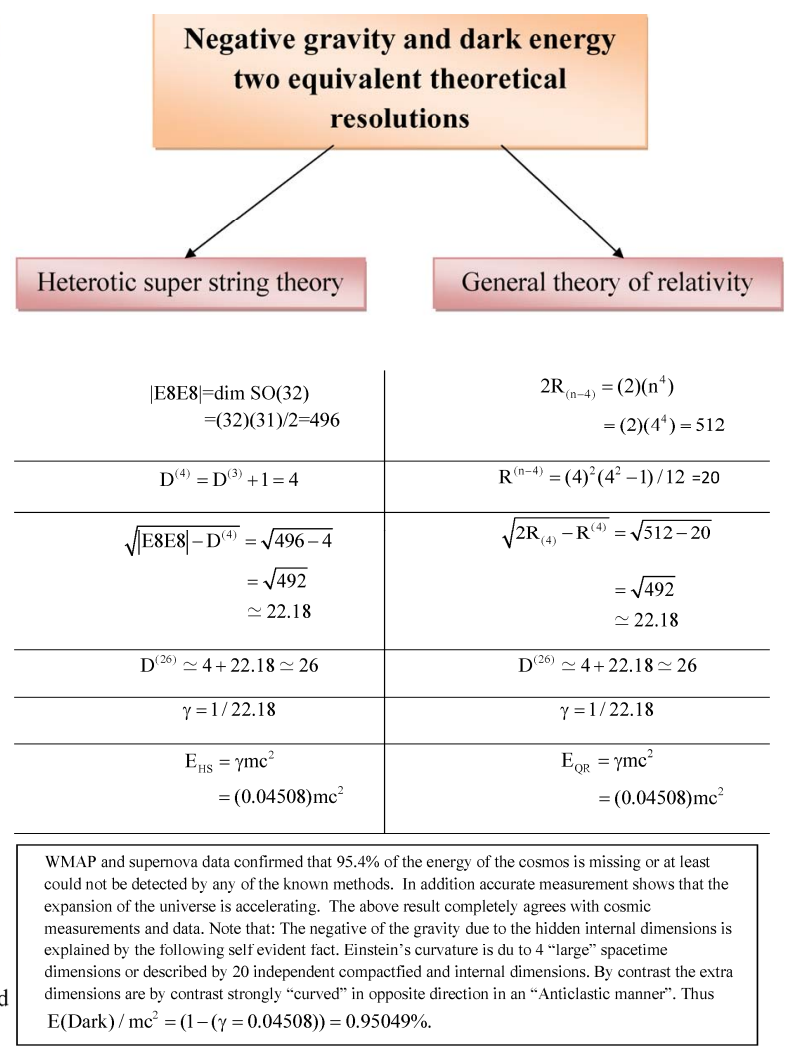

(b)

Figure 9. The deriving force behind gravity and negative gravity.

The fractal meaning of the constancy of the speed of light is explained in Figure 11 to round up the discussion and stress the fundamental role played by fractal geometry of spacetime in determining the duality of ordinary and dark energy [12-14,22,33].

\section{Analysis}

\subsection{The Speed of Light, Positive Energy, Negative Energy and Einstein's Energy}

Noting the result of point (2.5) we see that Einstein's equation may be viewed as based on an arithmetic average density or "volume" of 5D Kaluza-Klein space which does not differentiate between fractals due to voids and the voids in spacetime themselves and consequently Einstein's energy density is simply given by the famous equation based on continuous geometry and unit hyper volume equal to one $[12,26]$ :

$$
E(\text { Einstein })=(1)\left(m c^{2}\right)=m c^{2} .
$$

On the other hand ignoring the density of the voids and considering only the fractal part of spacetime represented by the five dimensional zero set, we then find the quantum relativity formula of ordinary energy density validated by direct cosmic measurement namely $[12,17$, 18] (see Figure 2)

$$
\begin{aligned}
& E_{Q R}=\left[\left(\phi^{5}\right) / 2\right] m c^{2}=m c^{2} /(22+k) \\
&=m c^{2} /(22.18033989) \quad m c^{2} / 22 \\
& \text { Ordinary Energy }(E(o))
\end{aligned}
$$

$=$ Energy of the quantum particle $=$ Positive Energy

Note that division by 2 in the above equation is due to arithmetic averaging and not due to using Newton's kinetic energy because in this particular interpretation velocity tends to be the velocity of light without multiplying it with $\phi$ as in unit interval physics using Sigalotti's critical value $[28,29]$. For an alternative derivation of $E_{O R}$ see Figures 3-8 as well as Figures 13 and 14.

By contrast if we consider spacetime voids only as represented by the quantum wave i.e. the empty set in 5D Kaluza-Klein theory (which means that it is the so-called dark energy of the cosmos), then we have the following complementary quantum relativity energy density formula: (see Figure 2 as well as Figures 13 and 14)

$$
\begin{aligned}
& E_{C Q R} \\
& =\left[\left(5 \phi^{2}\right) / 2\right] m c^{2}=m c^{2}\left(\frac{21+k}{22+k}\right) \quad m c^{2} /(21 / 22) \\
& =\text { Dark Energy }=\text { Energy of the quantum wave } \\
& =\text { Negative Energy }=\text { Halo Energy }
\end{aligned}
$$


Complexity

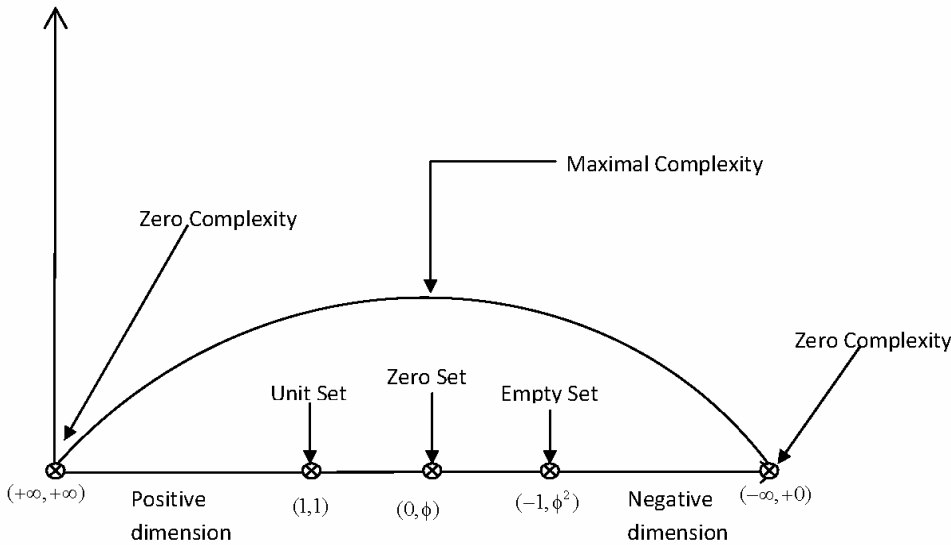

Complexity as function of negative and positive dimensions

The dimension of a set is given by two dimensions. The first is a topological and the second is a Hausdorff dimension. The $\mathrm{D}=(0, \phi)$ where $\phi=(\sqrt{5}-1) / 2$ is the dimension of the zero set. It may be surprising but it is extremely important to understand that at plus infinity $D=(+\infty,+\infty)$ as well as at minus infinity $\mathrm{D}=(-\infty, 0)$ we have Complexity equal to zero in both extremes. This have important consequence to absolute negative Kelvin temperature [41] as an aspect of Dark Energy related to negative and fractal dimensions. Note that the complementary curve to the above complexitydimensions curve is dual curve to that representing Entropy - Absolute temperature relationship for positive and negative Kelvin scale [41] as shown in fig. 7b. The plot may be also seen as a consequence of the unit interval physic of fig. (1) and Witten's T- duality of fig. $(3,4)$

(a)

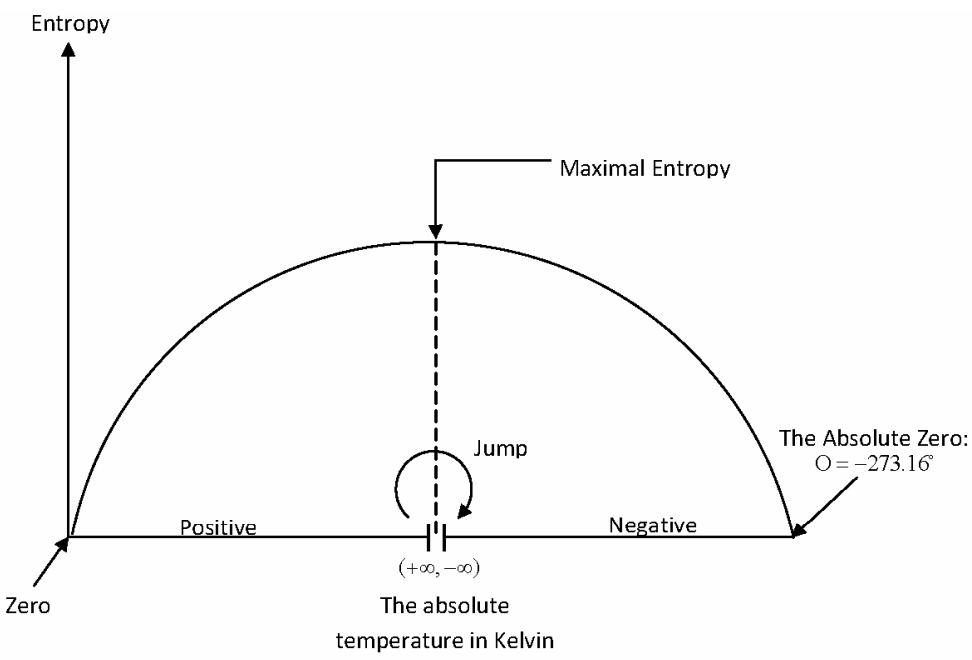

Entropy as function of positive and negative absolute temperature

This remarkable relation was investigated theoretically and experimentally in many pioneering efforts by various groups [41]. In the present work we stress the fact that this curve is dual to that of our complexity - dimension plot of fig. 7(a) and depends fundamentally on the notion of negative Menger - Uhryson topological dimensions used for the first time in this context by M.S. Elnaschie. This negative dimension leads to negative absolute temperature [41] and consequently to negative energy, negative gravity and thus Dark Energy and which explains the acceleration of the observed cosmic expansion.

(b)

Figure 10. (a) Complexity of maximal entropy and minimum entropy are equal; (b) Negative dimension and negative Kelvin temperature. 


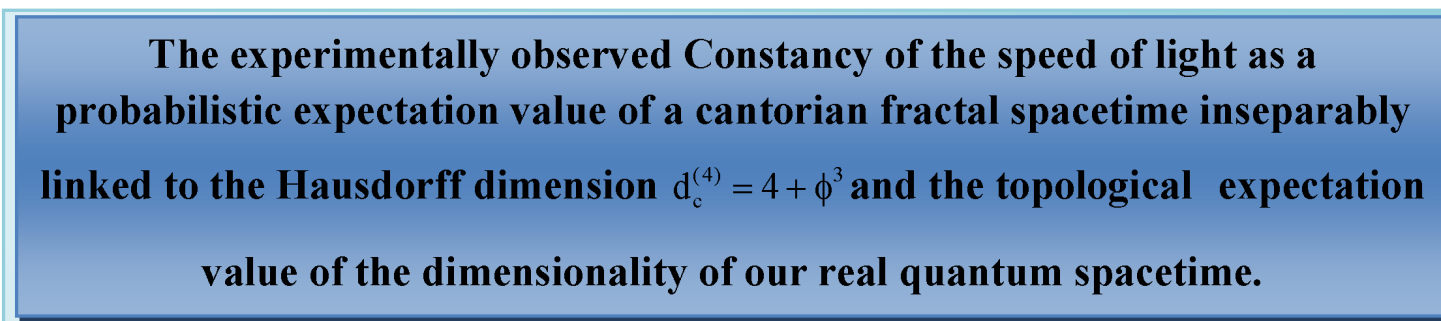

From Alain Connes' work on non commutative geometry [44] we know that

$\mathrm{D}_{4}=3+2 \phi=4+\phi^{2}$. Using E-Infinity this is given also by

$\mathrm{d}_{\mathrm{c}}^{(4)}=(1 / \phi)^{4-1}=(1 / \phi)^{3}=4+\phi^{3}$. This value is a probabilistic average or expectation

value. This is easily proven using the following center of gravity theorem of probability theory $[8,33]$

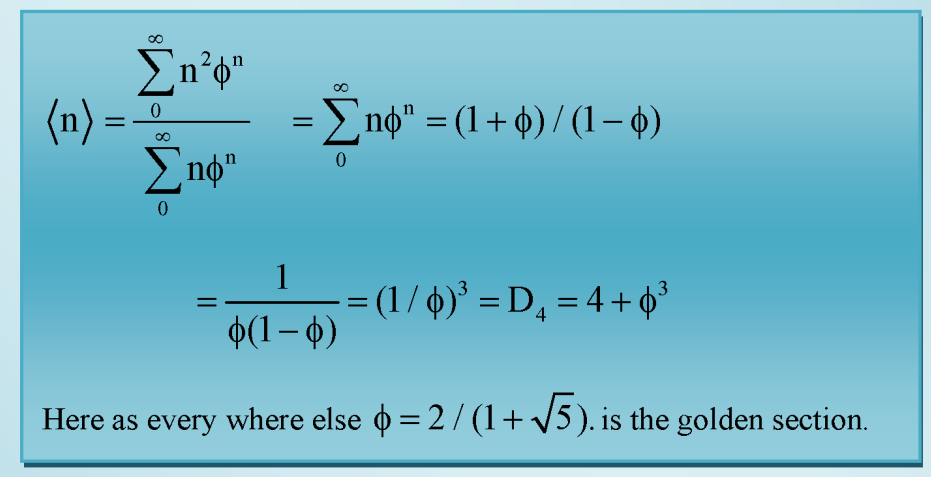

The constant speed of light is a similar expectation value. The "hidden" real speed of light varies between zero and infinity. In the topological dimension $D_{T}=4$ and Hausdorff dimension $\mathrm{D}_{\mathrm{H}}=4.236067$ we can observe only the average using direct experiments. The other spectrum of velocities can be inferred only indirectly via quantum effects such as Hardy's entanglement and requirements of quantum gravity.

Thus when $|\mathrm{c}|_{\max }=1$ this corresponds to when $\mathrm{c}=\infty$. This is similar to when $\bar{\alpha}_{\mathrm{QG}}=1$ for quantum gravity infinity strong Planck coupling which corresponds to maximum coupling. It may be seen as unit interval speed of light or Taiji topological speed of light which is the some as Sigalotti's critical value $\mathrm{c}=\phi$. Thus for dimensionless light velocity, the expectation value will correspond to $\phi$ and thus $4+\phi^{3}$ which means a topological dimension $\mathrm{D}_{\mathrm{T}}=4$. Consequently, as far as the speed of light is concerned we can measure it only indirectly in the $\mathrm{D}_{\mathrm{T}}=3+1$ dimension of classical space time and then we find that it is a phenomenological constant.

Figure 11. An overview regarding the constancy of the speed of light beeing a fractal mean value at the classical-quantum interface and thus constant.

where

$k=\phi^{3}\left(1-\phi^{3}\right)=2 \phi^{5}=0.18033989$ and $\phi=0.618033989$.

\subsection{Einstein's Energy Formula and a New Point of View}

From points discussed in the preceding sections we can conclude that Einstein's formula $E=m c^{2}$ is blind to the difference between ordinary energy i.e. quantum particle energy and dark energy i.e. the quantum wave energy because it presupposes a continuous smooth spacetime and never addresses any questions related to fractality or sparseness of spacetime which is the cause of the constancy of the speed of light at the classical interface being a fractal average (see Figure 11) [8,12,13,30]. However there are more profound consequences of the 


\section{The almost "Noble" number $\log (1+\sqrt{2})$ separating closed time-like curves from space-like curves in spinning Gödel universe and negative dark energy}

The spinning Gödel's universe proposed by Kurt Gödel in a 1949 paper dedicated to Albert Einstein with the irrelevant coordinate $z$ suppressed fallowing the exposition of S. Hawking and G. Ellis [40]. The space is rotationally symmetric about any point; the diagram represents correctly the rotational symmetry about the axis $\mathrm{r}=0$, and the time invariance. The light cone opens out and tips over as $\mathrm{r}$ increases (see line $\mathrm{L}$ ) resulting in closed time-like curves. The diagram does not correctly represent the fact that all points are in fact equivalent. However the vital point is that a noble number, namely the silver mean $1+\sqrt{2}$ scaled logarithmically $\log (1+\sqrt{2})$ appears in a remarkable way as a critical parameter separating time-like and space-like closed curves. This is reminiscent of two fundamental theories. First the Logarithmic scaling agrees with L. Nottale theory of scale relativity. Second $\log (1+\sqrt{2})$ is very close to the square of the golden mean $\phi^{2}$ as noted in a 2005 Chaos, Solitons \& Fractals article by El Naschie [40]. Thus $\phi^{2}$ may be regarded as a critical winding number similar to $\phi$ of KAM theorem of nonlinear dynamics where $\phi+\phi^{2}=1$. Equally noteworthy is that a quantum particle dual dimensional representation is the zero set $\mathrm{P}_{\mathrm{Q}} \equiv(0, \phi)$ while it's cobordism namely the empty set is nothing but the quantum wave $W_{Q} \equiv\left(-1, \phi^{2}\right)$ which the source of the dark energy. It is important to recall here that $P$. Davis found $\phi$ in the thermodynamics of black holes. We can therefore conjecture that a quantum particle is a mini black hole random cantor set with a Hausdorff dimension equal to the fractal weight of the photon namely $\phi=0.618033989$ and that we live in a clopen i.e. closed and opened universe which is its own multiverse. The maximal energy of a single particle equal in this universe is half of Hardy's quantum entanglement of two particles times $m c^{2}$ i.e. $E=\left(\phi^{5} / 2\right) \mathrm{cm}^{2}$ where $m$ is the mass, $c$ is the velocity of light and $\phi=2 /(1+\sqrt{5})$. Seen in this way we can conclude that Newton's kinetic energy $E_{N}=\frac{1}{2} m v^{2}$ and Einstein's formula $E=$ $\mathrm{mc}^{2}$ Could be fused together with Hardy's probability of quantum entanglement $\mathrm{p}=\phi^{5}$ to give quantum relativity EnergyMass relationship $E=\left(\phi^{5} / 2\right) m c^{2} \simeq m c^{2} / 22$ which explain fully the mystery of the missing dark energy of the Universe. Thus the quantum particle or the zero set is the source of ordinary measurable energy while the quantum wave or the empty set is behind the negative gravity of dark energy. We speculate that the intrinsic spinning of Godel universe could be an expression of the negative dark energy of the quantum wave i.e. the empty set [8].

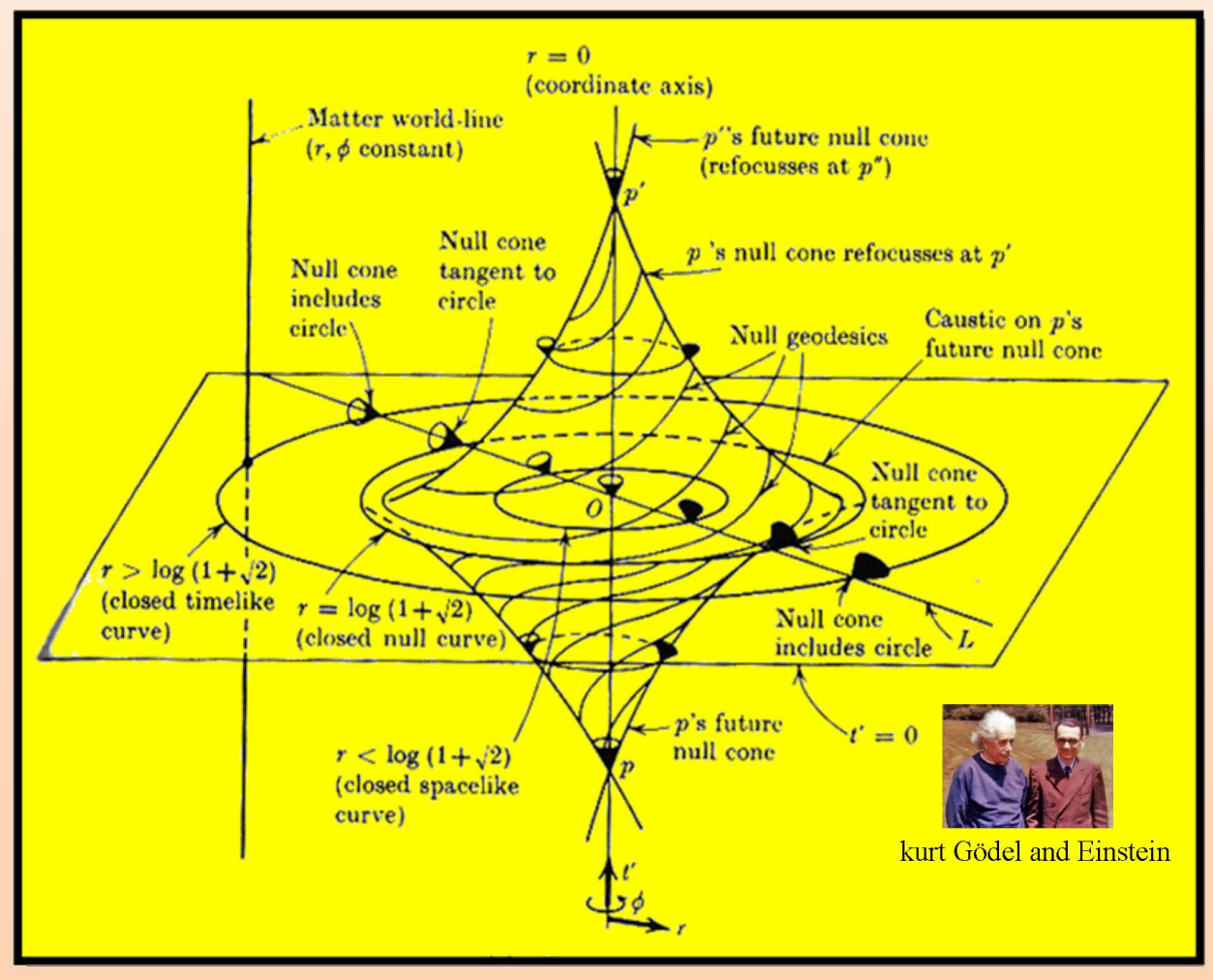

Figure 12. An overview regarding Gödel's universe. 


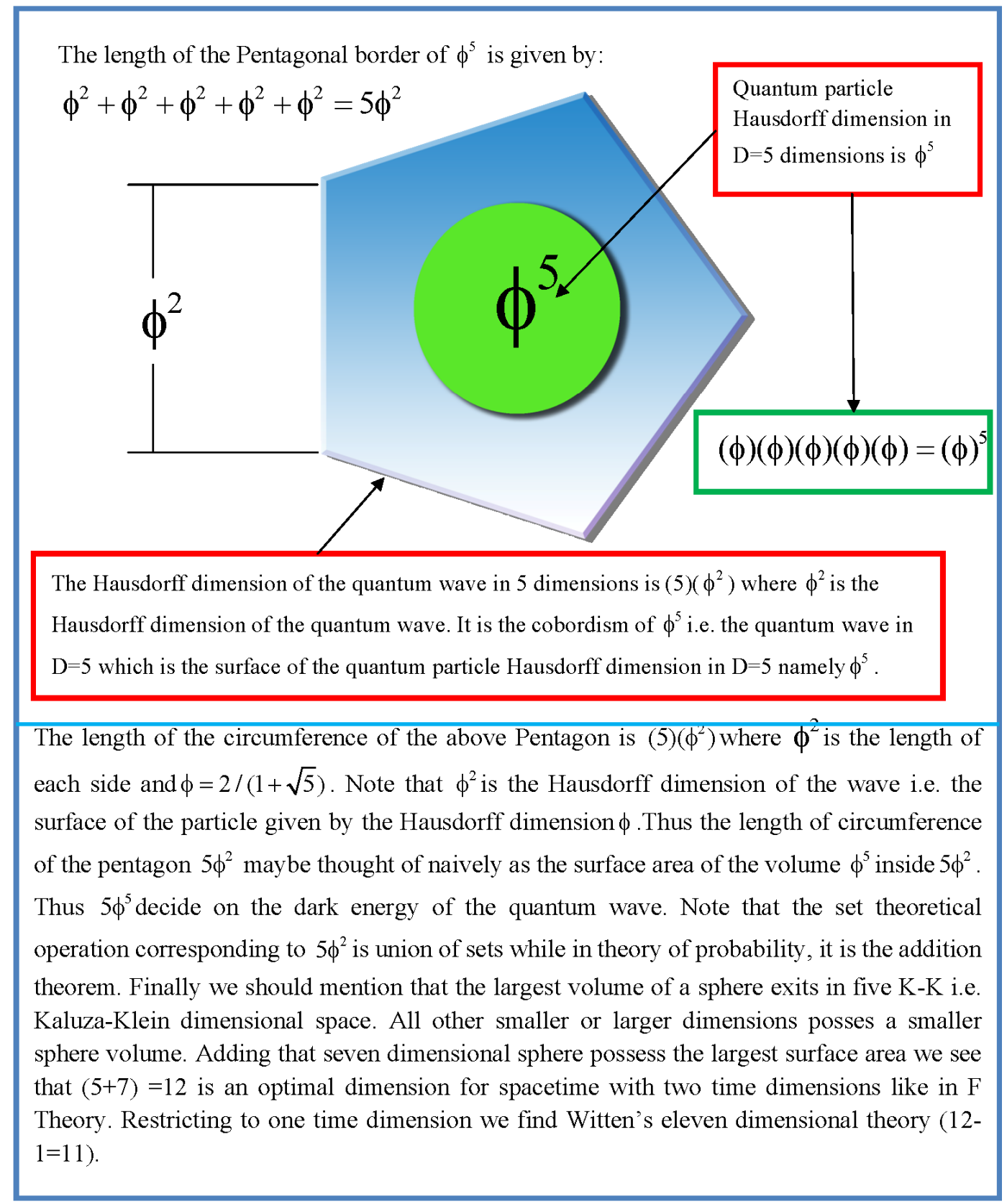

Figure 13. A naive geometrical interpretation of geometrical density or Hausdorff measure $\operatorname{vol}\left(D_{-1}\right)=5 \phi^{2}$ which decide upon the magnitude of dark energy of the quantum wave $E($ Dark $)=\frac{1}{2}\left(5 \phi^{2}\right) m c^{2}$.

spacetime voids than that which will be discussed in the next paragraph. For the moment we have only to recall that the wave is the cobordism i.e. the surface of the quantum particle and thus particle and wave are inseparable [8].

\subsection{The Negative Sign of the Quantum Wave Energy and Anti Gravity}

If we consider the effect of the two topological dimen- sions of the zero set and the empty set on our average pseudo Hausdorff Kaluza-Klein hyper volume or geometrical density then we could work out the following modified negative arithmetic average [8] (see Figures 1 and 2)

$$
\langle\operatorname{Vol}(\text { Total })\rangle=\frac{(o)\left(\phi^{5}\right)+(-1)\left(5 \phi^{2}\right)}{2}=-(5 \phi / 2)
$$

Consequently dark energy acquires in this case the 


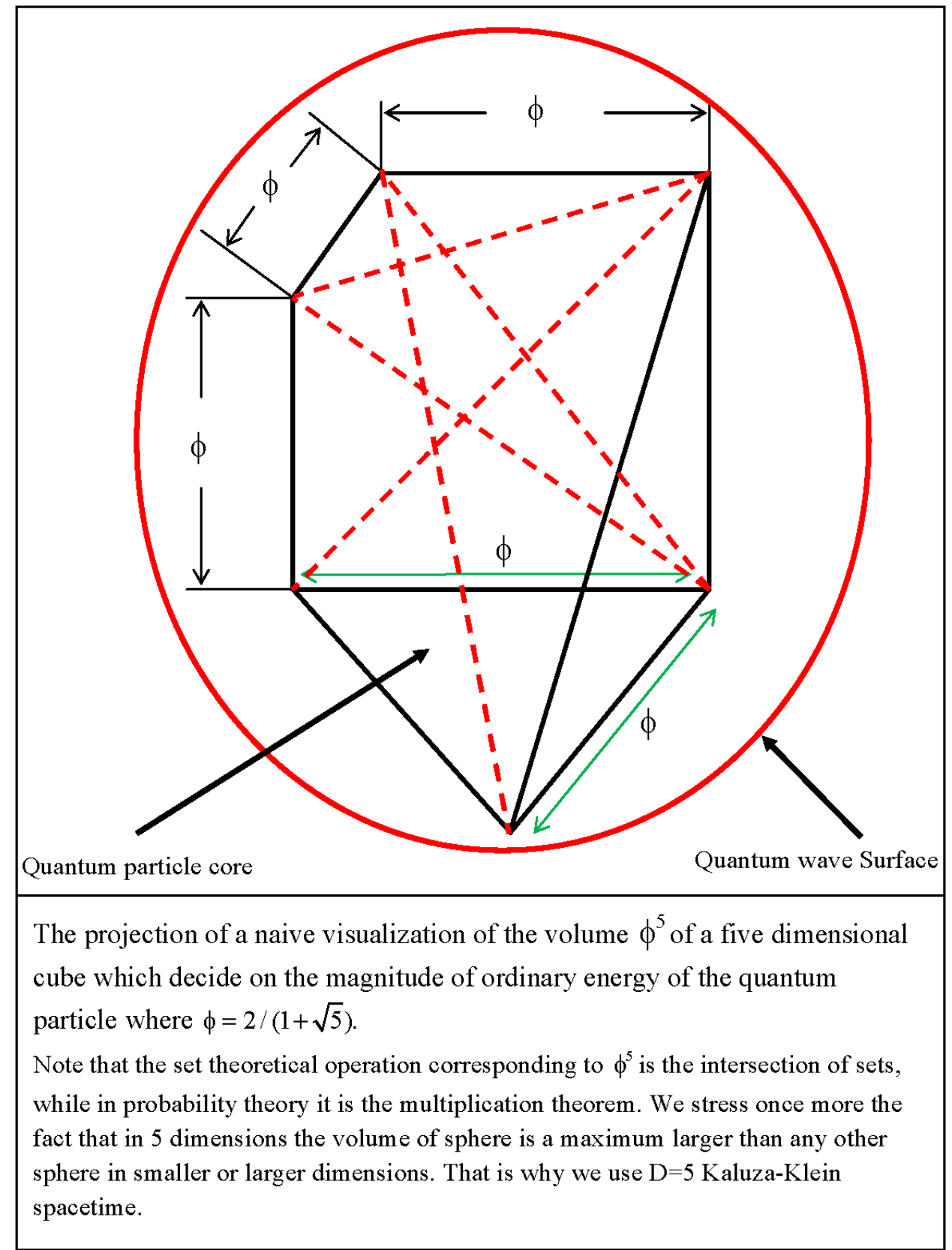

Figure 14. A naive geometrical interpretation of $\operatorname{vol}\left(D_{0}\right)=\phi^{2}$ of the ordinary energy of the quantum particle $E($ ordinary $)=\frac{1}{2}\left(\phi^{2}\right) m c^{2}$.

negative sign of the topological dimension of the empty set corresponding to the basic compactification equation of bosonic string theory $[2,6,17]$

$$
D^{(4)}-D^{(26)}=4-26=-22=D(\text { compactified })
$$

This negative sign (see Figure 9) is therefore an additional indication that dark energy or what is equivalently the energy of the quantum wave i.e. the empty set differs fundamentally from ordinary energy for being negative and represents effectively negative gravity pushing in the opposite direction of classical gravity and causing the cosmic expansion of the Universe to accelerate rather than de-accelerate in agreement with relatively recent accurate observations [9-17].

\section{Intermediate Discussion}

We conclude that while fractals i.e. the zero set or phenomenologically the quantum particle is behind ordinary 
energy, voids in spacetime corresponding to the empty set i.e. the quantum wave i.e. the halo of the quantum particle $[8,23,33,44]$ are not only behind dark energy but also are the cause of negative gravity and the increased rate of cosmic expansion [9-11,15,16]. Negative gravity is essentially due to negative curvature of spacetime. If we see gravity as the Riemannian curvature of $D^{(4)}=4$ spacetime, then negative curvature [9] is related to $D($ compactified $)=-22$ and is akin to the anticlastic curvature (see Figure 9) known from the behavior of elastic materials and theories of elasticity and plasticity [35-37]. A torsion tensor like in Cosserat micropolar media and similar classical field theories may actually simulate something similar to Cartan relativity and admit negative curvature and antigravity [2]. Thus we have here the intuitive phenomenological explanation arising from the effect of Poisson's ratio that when a long thick beam [35-37] is curved in the longitudinal direction then cross section curves in the opposite perpendicular direction [35]. Consequently, we could say that $D=-22$ has the effect of an anti-curvature producing anti-gravity [9] due to negative energy of the halo [9] of the quantum particle i.e. the quantum wave which we have dubbed dark energy for want of a better physical word communicating the deep meaning [9-18]. We can go even further still and claim that negative absolute temperature is another physical manifestation of negative dimensions and the empty set (see Figure 10) and therefore negative energy and consequently dark energy [41]. The epistemological question of how a wave devoid of ordinary energy could induce a measurable negative gravity effect is essentially the other side of the fact that this same wave decides on the location of a quantum particle as confirmed by theory and experiment [1-3]. In other words the quantum probability wave possesses an ontological quality. Said differently we know that measurement collapses the quantum wave by converting an empty set particle to zero set wave. Consequently cosmic measurement collapses the Hawking-Hartle wave solution of the Wheeler-DeWitt Schrödinger equation of the universe and therefore we cannot see or measure any energy of this wave unless we develop quantum nondemolition measurement instrument [1].

\section{Various Interpretations and Duality in Five Dimensional Kaluza-Klein Spacetime}

Ordinary energy density and dark energy density are two dual yet complementary faces to a one common energy given by the sum of both which is equal to Einstein's famous equation $E=m c^{2}$ [2]. Unlike ordinary energy which is entirely rooted in the zero set describing a generic quantum particle state, dark energy is solely due to the quantum wave aspect of the quantum particle as modeled by the empty set [8]. Nothing could show this symmetric duality better than writing both types of ener- gies in the Magueijo-Smolin formalism [5,26] and their elegant energy expression (see Figure 3):

$$
E=\frac{m c^{2}}{1+\frac{m c^{2}}{E_{P}}} .
$$

Here $m$ is the five dimensional Kaluza-Klein mass [38,39], $c$ is the speed of light and $E_{P}$ is the Planck energy. Using the unit interval set theoretical value for $m c^{2} / E_{P}$ it was reasoned earlier on that we must have: $m=5, c^{2}=\phi^{2}$ and $E_{P}=\phi^{5}$ and inserting in $E$ we find that $[12,17]$ (see Figure 3)

$$
\begin{aligned}
E & =\frac{m c^{2}}{1+(21+k)}=\frac{m c^{2}}{22+k} \\
& =\left(\phi^{2} / 2\right)\left(m c^{2}\right) \quad m c^{2} / 22=E(\text { Ordinary })
\end{aligned}
$$

exactly as should be. However using Witten's T-duality [2,4,6,40] (see Figure 3):

$$
\frac{m c^{2}}{E_{P}} \stackrel{T}{\longleftrightarrow} \frac{E_{P}}{m c^{2}}
$$

and inserting in $E$ one finds

$$
\begin{aligned}
E & =\frac{m c^{2}}{1+\frac{1}{21+k}}=\left(m c^{2}\right)(21+k) /(22+k) \\
& =\left(5 \phi^{2} / 2\right)\left(m c^{2}\right) \quad\left(m c^{2}\right)(21 / 22)=E(\text { Dark }) .
\end{aligned}
$$

where $k=\phi^{3}\left(1-\phi^{3}\right)=0.18033989 \quad[22,33]$.

Thus we have demonstrated in the present work and presumably for the first time that dark energy is essentially the absolute value of the negative energy density of the Hawking-Hartle quantum wave solution of Feynman-Wheeler-DeWitt Schrödinger equation of the entire universe and as such it induces the hitherto unknown phenomena of negative gravity which we and others consider to be the cause of the observed increased rate of cosmic expansion [9-18]. This physical interpretation makes a great deal of sense because the set theoretical definition of the quantum wave is the empty set represented by $\operatorname{Dim}($ empty set $) \equiv\left(-1, \phi^{2}\right)$ whose topological dimension is minus one $(-1)$. Consequently the accurate way to write down the quantum wave energy density is $[8,9,32]$

$$
\begin{gathered}
|E|=\left|-\left(5 \phi^{2} / 2\right)\left(m c^{2}\right)\right| \\
\left|-m c^{2}(21 / 22)\right|
\end{gathered}
$$

where 5 is the dimension of Kaluza-Klein spacetime [42, 43]. To gain an even deeper understanding of the broken symmetry and duality between particle and wave and consequently between ordinary energy and negative or dark energy, let us start this time from Newton's classical 
expression for the kinetic energy [2]

$$
E_{N}=\frac{1}{2} m v^{2} .
$$

Recalling the set theoretical duality between the quantum particle zero set and the quantum wave empty set [8]

$$
0 \leftrightarrow-1 \text { and } \phi \leftrightarrow \phi^{2}
$$

as well as the dual transformation given for particle and wave as in the following box:

Box No. 1. Particle-wave duality transformation.

\begin{tabular}{cl}
\hline For particle & For wave \\
\hline$m \rightarrow \phi^{3} m$ & $m \rightarrow 5 m$ \\
$v \rightarrow \phi c$ & $v \rightarrow \phi c$ \\
\hline
\end{tabular}

Then inserting in $E_{N}$ one finds the following particle and wave energy as per the next box:

Box No.2. Quantum particle ordinary energy and quantum wave dark energy.

\begin{tabular}{cr}
\hline For particle & For wave \\
\hline$E_{N} \rightarrow E=\frac{1}{2} \phi^{3} m \phi^{2} c^{2}$ & $E_{N} \rightarrow E=\frac{1}{2} 5 m \phi^{2} c^{2}$ \\
$=\left(\phi^{5} / 2\right) m c^{2}$ & $=\left(5 \phi^{2} / 2\right) m c^{2}$ \\
$m c^{2} / 22$ & $m c^{2}(21 / 22)$ \\
$=$ Ordinary Positive Energy & $=$ Dark Energy \\
\hline
\end{tabular}

Let us contemplate the meaning of $\phi^{3}$ in $\phi^{3} m$ and 5 in $5 \mathrm{~m}$ as well as $\phi$ in $\phi c$. The simplest way to explain it in the present context is $\phi$ in $\phi c$. This stems from Sigalotti's critical speed given by $v / c=\phi$. The $\phi^{3}$ on the other hand is $\phi^{3}=1 /\langle n\rangle=1 /\left(4+\phi^{3}\right)$ where $\langle n\rangle=4+\phi^{3}$ is the Hausdorff dimension of a four dimensional set given by $\left(4 ; 4+\phi^{3}\right)$ and representing the core of fractal-Cantorian spacetime [21,22]. At the same time $\phi^{3}$ is the global quantum entanglement probability or what some call the counter factual effect of the spacetime surrounding a quantum particle [32]. It is numerically equal to the Unruh dimensionless thermal temperature [2] and together with $\phi^{2}$ gives $\left(\phi^{3}\right)\left(\phi^{2}\right)=\phi^{5}$ which is glement of two quantum particles [37]. On the other hand the appearance of 5 in $5 \mathrm{~m}$ maybe seen as the five dimensionality of a Kaluza-Klein spacetime $[2,4,6]$. This is an ordinary 4D-Einstien space with a single additional cyclical pipe-like compactified extra dimension joining electro magnetism to gravity $[38,39,42,43]$ (see Figure 4). It could be also seen as an extra spin half fermionic dimension [22]. However like the bosonic fractal dimension $4+\phi^{3}$ enters into the energy equation of the quantum particle in the inverse form $1 /\left(4+\phi^{3}\right)=\phi^{3}$, the non-fractal five dimensional fermionic $\mathrm{K}-\mathrm{K}$ spacetime
$[38,39]$ enters into the energy equation of the quantum wave or dark energy directly simply as factor 5 . For the sake of completeness let us see what would result from considering a fractal i.e. a transfinitely quantized KleinKaluza spacetime developed by the Author [38,39, 42,43] with $5 \rightarrow 5+\phi^{3}$ which means (see Figure 4)

$$
5+\phi^{3}=5+\frac{1}{4+\frac{1}{4+\frac{1}{4+}}} .
$$

In this case $\left(5+\phi^{3}\right)\left(\phi^{2}\right)$ would mislead us into wrongly thinking that dark energy is equal to Einstein's energy (see Figures 1 and $\mathbf{4}$ )

$$
\begin{aligned}
& E=\left(5+\phi^{3}\right)\left(\phi^{2}\right)\left(\frac{1}{2}\right) m c^{2} \\
& =\left(5 \phi^{2} / 2\right) m c^{2}+\left(\phi^{3} \phi^{2} / 2\right) m c^{2} \\
& =\text { Ordinary Energy }+ \text { Dark Energy } \\
& =\text { Particle Position Energy + Wave kinetic Energy } \\
& =(2)(1 / 2) m c^{2}=\text { Total Potential Energy } \\
& =m c^{2}=E(\text { Einstein })
\end{aligned}
$$

This is initially a surprising result but it is a misunderstanding as well because it shows that $\mathrm{E}$ (Einstein) is obtainable from a fractal five-dimension Kaluza-Klein spacetime while dark energy is the child of a mere fivedimensional non-fractal Kaluza-Klein spacetime. However the difference is namely that $\phi^{3}$ is the factor responsible for the additional energy which is nothing but the ordinary energy which when added to non-fractal 5D Klein Kaluza [38,39] i.e. to dark energy gives the total energy, namely that of Einstein's energy. This confirms all of our earlier results namely that $E=m c^{2}$ could be obtained via either a smooth $4 \mathrm{D}$ spacetime or via a delicate analysis using a fractal $D=5+\phi^{3}$ five dimension spacetime (see Figure 4). Seen that way $D=5+\phi^{3}$ which is related to the fractal 11-D theory via $D=11+\phi^{5}$ is a more fundamental theory than Einstein's $D=4$ spacetime theory. In fact we could deduce $D=5+\phi^{3}$ from $D=11+\phi^{5}$ by dividing by 2 and subtracting the number weight of 1 photon [20] namely $\phi$ so that one finds (see Figure 4)

$$
\left[\left(11+\phi^{5}\right) / 2\right]-(\gamma=\phi)=5+\phi^{3}
$$

In other words we can either interpret $\phi^{5}$ in $E_{1}$ as a hyper volume or Hausdorff measure and division by 2 as averaging or we see it as the transformation $m \rightarrow \phi^{3} m$ and $v \rightarrow \phi c$ and division by 2 as coming from using Newton's kinetic energy.

We conclude this section by stressing that there are three and not only one Kaluza-Klein theories. The first is 
the classical 5D theory of Kaluza [41]. The second is the quantized 5D theory of Klein $[41,42]$. The third is the transfinitely quantized $\left(5+\phi^{3}\right) D$ theory developed by the author $[38,39,43]$.

\section{Conclusions}

Dark energy is a non-classical form of negative energy density stemming from the quantum wave empty set characteristic and we found it quantitatively to be given by the absolute value of

$$
E(\text { dark })=\left|-\left(5 \frac{\phi^{2}}{2}\right)\left(m c^{2}\right)\right| \quad m c^{2}(21 / 22)
$$

where $\phi^{2}$ could be interpreted as the local Hardy's type quantum entanglement of two particles or the Hausdorff dimension of the empty set and $\phi=2 /(\sqrt{5}+1)$.

Ordinary energy on the other hand is given by the positive energy of the zero set describing the quantum particle and found in the present work to be equal [12]

$$
E(\text { ordinary })=\left(\phi^{5} / 2\right)\left(m c^{2}\right) \quad m c^{2} / 22
$$

where $\phi^{5}$ is the famous golden mean Hardy's quantum entanglement $[2,31]$.

There are numerous other methods and theories which lead to essentially the same conclusion regarding ordinary positive energy density being equal to Einstein's energy divided by 22 which are summarized in Figures 5-8. In particular Figure 7 shows how to derive $\phi^{5} / 2 \quad 1 / 22$ from Nash's embedding plus the notion of spacetime sparseness and Figure 8 gives an idea about the role of bosonic strings dimension.

A particular tantalizing result is that the sum of both energies, dark energy and ordinary energy, is a neat confirmation of Einstein's famous relativity formula because

$$
\begin{aligned}
E & =\left(5 \phi^{5} / 2\right) m c^{2}+\left(\phi^{5} / 2\right) m c^{2} \\
& =\left(m c^{2}\right)(1)=m c^{2}=E(\text { Einstein }) .
\end{aligned}
$$

The negatives of the dark energy on the other hand confirm earlier string theoretical conjectures that negative gravity is due to negative energy of the compactified 22 dimensions which produce negative curvature as per the bosonic string dimensional equation $[2,4,12]$

$$
D^{(4)}-D^{(26)}=4-22=D^{(22)}=-22 \text {. }
$$

Following the same reasoning one can write the $(21 / 22)$ factor of dark energy as

$$
\frac{D^{(5)}-D^{(26)}}{D^{(4)}-D^{(26)}}=\frac{5-22}{4-22}=\frac{-21}{-22}=(21 / 22)
$$

which proves that $(1 / 22)$ of ordinary energy and $(21 / 22)$ of dark energy have opposite signs in any event. In fact the present theory strongly suggests the physical reality of Kaluza-Klein extra fifth dimension $[42,43]$ as well as the fractality of spacetime and ontological reality of the quantum wave $[8,13,14,20,43,44]$ all apart from the reality of dark energy which we cannot see or measure because measurement collapses the quantum wave.

For a different view of negative gravity via general relativity and comparison with Heterotic string theory see Figure 9 and commentary therein.

It is worth noting that negative dimensions are behind the experimentally observed negative Kelvin absolute temperature [41] and may thus be looked upon as another physical interpretation of dark energy (see Figures 10(a) and (b)). Furthermore if the building blocks of spacetime are random Cantor sets as the present work suggests, then our cosmos is clopen i.e. opened and closed because Cantor sets are clopen.

We could understand the repelling effect of dark energy on a cosmic scale as a phenomenon basically due to the fact that the motion of quantum objects and its spatial location is determined completely by the quantum wave following prescription [1-3]. It is a matter of speculation that the spinning of Gödel's universe (see Figure 12) [40] is related to dark energy and repelling gravity. Finally the constancy of the speed of light as a fractal average is explained in some detail in Figure 11.

\section{REFERENCES}

[1] S. Nakajma, et al., "Foundation of Quantum Mechanics in the Light of New Technology," World scientific, Singapore, 1996.

[2] R. Penrose, "The Road to Reality," Jonathan Cape, London, 2004.

[3] D. R. Finkelstein, "Quantum Relativity," Springer, Berlin, 1996. doi:10.1007/978-3-642-60936-7

[4] M. Duff, "The World in Eleven Dimensions," IOP Publishing, Bristol, 1999.

[5] J. Mageuijo and L. Smolin, "Lorentz Invariance with an Invariant Energy Scale," Cornell University Library, Ithaca, 2001.

[6] J. Polchinski, "String Theory. Vol. I and II," Cambridge University Press, Cambridge, 1999.

[7] C. Rovelli, "Quantum Gravity," Cambridge Press, Cambridge, 2004.

[8] J.-H. He and M. S. El Naschie, "On the Monadic Nature of Quantum Gravity as Highly Structured Golden Ring Spaces and Spectra," Fractal Spacetime and Noncommutative Geometry in Quantum and High Energy Physics, Vol. 2, No. 2, 2012, pp. 94-98.

[9] E. J. Copeland, M. Sami and S. Tsujikawa, "Dynamics of Dark Energy," Cornell University Library, Ithaca, 2006.

[10] L. Amendola and S. Tsujikawa, "Dark Energy Theory and Observations," Cambridge University Press, Cambridge, 2010. doi:10.1017/CBO9780511750823

[11] S. Perlmutter, et al. (Supernova Cosmology Project Col- 
laboration), "Measurements of Omega and Lambda from 42 High-Redshift Supernova," The Astrophysical Journal, Vol. 517, No. 2, 1999, pp. 565-585. doi:10.1086/307221

[12] M. S. El Naschie and L. Marek-Crnjac, "Deriving the Exact Percentage of Dark Energy Using a Transfinite Version of Nottale's Scale Relativity," International Journal Of Modern Nonlinear Theory and Applications, Vol. 1, No. 4, 2012, pp. 118-124.

[13] Y. Baryshev and P. Teerikorpi, "Discovery of Cosmic Fractals," World Scientific, Singapore, 2002.

[14] L. Nottale, "Scale Relativity," Imperial College Press, London, 2011.

[15] Planck-Spacecraft.Wikipedia. http://en.wikipedia.org/wiki/Planck. 15/09/2012. 2012.

[16] R. Panek, "Dark Energy: The Biggest Mystery in the Universe," The Smithsonian Magazine. http://www.smithsonianmag.com/science-nature/Dark-En ergy-The-Biggest-Mystery-in-the-Universe.html

[17] M. S. El Naschie, "Revising Einstein's $E=m c^{2}$. A Theoretical resolution of the mystery of dark energy," Conference Program and Abstracts of the Fourth Arab International Conference in Physics and Material Sciences, Bibliotheca Alexandrina, Alexandria, 2012, p. 1.

[18] J.-H. He, "A Historical Scientific Finding on Dark Energy by M. S. El Naschie," International Symposium on Nonlinear Dynamics, Suzhou \& Shanghai, 27-30 October 2012. See also the Journal Fractal Spacetime and NonCommutatitve Geometry in Quantum and High Energy Physics, Vol. 2, No. 2, 2012, p. 154.

[19] M. S. El Naschie, L. Marek-Crnjac and J.-H. He, "On the Mathematical Philosophy of Being and Nothingness in Quantum Physics," Fractal Spacetime and Noncommutative Geometry in Quantum and High Energy Physics, Vol. 2, No. 2, 2012, pp. 103-106.

[20] M. S. El Naschie, J.-H. He, S. I. Nada, L. Crnjac and M. A. Helal, "Golden Mean Computer for High Energy Physics," Fractal Spacetime and Noncommutative Geometry in Quantum and High Energy Physics, Vol. 2, No. 2, 2012, pp. 80-93.

[21] M. S. El Naschie, "Elementary Prerequisites for E-Infinity (Recommended Background Readings in Nonlinear Dynamics, Geometry and Topology)," Chaos, Solitons \& Fractals, Vol. 30, No. 3, 2006, pp. 579-605. doi:10.1016/j.chaos.2006.03.030

[22] M. S. El Naschie, "A Review of E-Infinity and the Mass Spectrum of High Energy Particle Physics," Chaos, Solitons \& Fractals, Vol. 19, No. 1, 2004, pp. 209-236. doi:10.1016/S0960-0779(03)00278-9

[23] M. S. El Naschie, M. A. Helal, L. M. Crnjac and J.-H. He, "Transfinite Corrections as a Hardy Type Quantum Entanglement," Fractal Spacetime and Noncommutative Geometry in Quantum and High Energy Physics, Vol. 2, No. 2, 2012, pp. 99-102.

[24] W. Rindler, "Relativity, Special, General and Cosmological," Oxford Press, Oxford, 2004.

[25] J.-P. Hsu and L. Hsu, "A Broad View of Relativity," World Scientific, Singapore, 2006.
[26] J. Mageuijo, "Faster Than the Speed of Light," William Heinemann, London, 2003.

[27] H. Saller, "Operational Quantum Theory, Vol. I \& II," Springer, Berlin, 2006.

[28] L. Sigalotti and A. Mejias, "The Golden Mean in Special Relativity," Chaos, Solitons \& Fractals, Vol. 30, No. 3, 2006, pp. 521-524. doi:10.1016/i.chaos.2006.03.005

[29] S. Hendi and M. S. Zadeh, "Special Relativity and the Golden Mean," Journal of Theoretical Physics, Vol. 1, 2012, pp. 37-45.

[30] M. S. El Naschie, "On a Class of Fuzzy Kähler-Like Manifolds," Chaos, Solitons \& Fractals, Vol. 26, No. 2, 2005, pp. 257-261. doi:10.1016/j.chaos.2004.12.024

[31] J.-H. He, et al., "Quantum Golden Mean Entanglement Test as the Signature of the Fractality of Micro Spacetime," Nonlinear Science Letters B, Vol. 1, No. 2, 2011, pp. 45-50.

[32] M. S. El Naschie, "Quantum Entanglement as a Consequence of a Cantorian Micro Spacetime Geometry," Journal of Quantum Information Science, Vol. 1, No. 2, 2011, pp. 50-53. http://www.scirp.org/journal/jqis/

[33] M. S. El Naschie, "The Theory of Cantorian Spacetime and High Energy Particle Physics (An Informal Review)," Chaos, Solitons \& Fractals, Vol. 41, No. 5, 2009, pp. 2635-2646. doi:10.1016/j.chaos.2008.09.059

[34] M. S. El Naschie, "The Discrete Charm of Certain Eleven Dimensional Spacetime Theory," International Journal of Nonlinear Sciences and Numerical Simulation, Vol. 7, No. 4, 2006, pp. 477-481.

[35] D. Horrocks and W. Johnson, "On Anticlastic Curvature with Special Reference to Plastic Bending," International Journal of Mechanical Sciences, Vol. 9, No. 12, 1967, pp. 835-844.

[36] M. S. El Naschie, "Stress, Stability and Chaos in Structural Engineering," McGraw Hill, London, 1990.

[37] W. Koiter, "Elastic Stability and Post Buckling Behaviour in Nonlinear Problems," University of Wisconsin Press, Maidison, 1963.

[38] M. S. El Naschie, "Kaluza-Klein Unification Same Possible Extinctions," Chaos, Solitons \& Fractals, Vol. 37, 2008, pp. 16-22.

[39] M. S. El Naschie, "On Dualities between NordstromKaluza-Klein, Newtonian and Quantum Gravity," Chaos, Solitons \& Fractals, Vol. 36, 2009, pp. 808-810.

[40] M. S. El Naschie, "Gödel Universe, Dualities and High Energy Particles in E-Infinity," Chaos, Solitons \& Fractals, Vol. 25, No. 3, 2005, pp. 759-764. doi:10.1016/j.chaos.2004.12.010

[41] J. Aron, "Cloud of Atoms Goes beyond Absolute Zero," New Scientist, Vol. 217, No. 2899, 2013, p. 12. doi:10.1016/S0262-4079(13)60081-0

[42] P. Halpern, "The Great beyond, Higher Dimensions, Parallel Universes and the Extra Ordinary Search for a Theory of Everything," John Wiley, New Jersey, 2004.

[43] M. S. El Naschie, "A Tale of Two Klein's Unified in Strings and E-Infinity Theory," Chaos, Solitons \& Frac- 
tals, Vol. 26, No. 1, 2005, pp. 247-254.

doi:10.1016/j.chaos.2005.01.016

[44] A. Connes, "Alain Connes Noncommutative Geometry,"
Academic Press, New York, 1994, see in Particular pp. 88-93. 